\title{
A NOTE ON THE MEAN VALUE OF THE POISSON KERNEL
}

\section{A. S. GALBRAITH AND J. W. GREEN}

In some investigations it is necessary to evaluate the mean value of some power of the Poisson kernel,

$$
P(r, \theta) \equiv\left(1-r^{2}\right) /\left(1-2 r \cos \theta+r^{2}\right),
$$

with respect to $\theta$. This note gives a closed expression for this mean value, and an exact statement of the order of growth as $r$ approaches 1 .

Theorem 1. If $x=2 r /\left(1+r^{2}\right)$, then

$$
\begin{aligned}
\frac{1}{2 \pi} \int_{0}^{2 \pi} P^{n+1}(r, \theta) d \theta= & \left(\frac{1-r^{2}}{1+r^{2}}\right)^{n+1} \cdot \frac{1}{\Gamma(n+1)} \\
& \cdot \frac{d^{n}}{d x^{n}}\left(\frac{x^{n}}{\left(1-x^{2}\right)^{1 / 2}}\right), \quad n>-1 .
\end{aligned}
$$

If $n$ is not an integer the derivative is to be computed by the formula of Riemann and Liouville ${ }^{1}$

$$
\frac{d^{n}}{d x^{n}}(f(x))=\frac{d^{m}}{d x^{m}} \frac{1}{\Gamma(\rho)} \int_{0}^{x}(x-t)^{\rho-1} f(t) d t,
$$

where $m$ is the smallest integer not less than $n$ and $\rho=m-n$.

The proof consists merely of the comparison of two power series. Clearly

$$
P^{n+1}(r, \theta)=\left(\frac{1-r^{2}}{1+r^{2}}\right)^{n+1}\left(1-\frac{2 r}{1+r^{2}} \cos \theta\right)^{-(n+1)},
$$

and the second parenthesis, with $x=2 r /\left(1+r^{2}\right)$, is $1+(n+1) x \cos \theta$ $+(n+1)(n+2) / 2 ! x^{2} \cos ^{2} \theta+\cdots$ by the binomial theorem. Since

$$
\begin{aligned}
\int_{0}^{2 \pi} \cos ^{p} \theta d \theta & =0 \quad \text { (if } p \text { is an odd integer) } \\
& =\frac{4(p-1)(p-3) \cdots 3 \cdot 1}{p(p-2) \cdots 4 \cdot 2} \cdot \frac{\pi}{2} \text { (if } p \text { is even) }
\end{aligned}
$$

Received by the editors October 28, 1946.

${ }^{1}$ See, for example, Courant, Differential and integral calculus, rev. ed., vol. 2, pp. 339-340. 
equation (1) can be written

(3)

$$
\begin{aligned}
\frac{1}{2 \pi} \int_{0}^{2 \pi} & P^{n+1}(r, \theta) d \theta \\
= & \left(\frac{1-r^{2}}{1+r^{2}}\right)^{n+1}\left[1+\frac{(n+1)(n+2)}{2 !} \cdot x^{2} \cdot \frac{1}{2}\right. \\
& \left.+\frac{(n+1)(n+2)(n+3)(n+4)}{4 !} \cdot x^{4} \cdot \frac{1 \cdot 3}{2 \cdot 4}+\cdots\right] \\
= & \left(\frac{1-r^{2}}{1+r^{2}}\right)^{n+1}\left[1+\sum_{k=1}^{\infty} \frac{(n+1)(n+2) \cdots(n+2 k)}{(2 k) !}\right. \\
& \left.\frac{1 \cdot 3 \cdot 5 \cdots(2 k-1)}{2^{k} \cdot k !} \cdot x^{2 k}\right] .
\end{aligned}
$$

Now

$$
\begin{aligned}
x^{n}\left(1-x^{2}\right)^{-1 / 2}=x^{n} & +\frac{1}{2} x^{n+2}+\frac{1 \cdot 3}{2^{2} \cdot 2 !} x^{n+4} \\
& +\frac{1 \cdot 3 \cdot 5}{2^{3} \cdot 3 !} x^{n+6}+\cdots
\end{aligned}
$$

If $n$ is an integer, this can be differentiated $n$ times to yield

$$
\begin{aligned}
n ! & +\frac{(n+2) !}{2 !} \cdot \frac{1}{2} \cdot x^{2}+\frac{(n+4) !}{4 !} \cdot \frac{1 \cdot 3}{2^{2} \cdot 2 !} x^{4} \\
& +\frac{(n+6) !}{6 !} \cdot \frac{1 \cdot 3 \cdot 5}{2^{3} \cdot 3 !} x^{6}+\cdots
\end{aligned}
$$

Division by $\Gamma(n+1)$, as required in (1), produces the power series of (3).

If $n$ is not an integer, formula (2) is applied to (4) to yield, on the right, a series of terms containing integrals of the form

$$
\frac{1}{\Gamma(\rho)} \int_{0}^{x}(x-t)^{\rho-1} t^{n+2 p} d t, \quad p=0,1,2, \cdots .
$$

The substitution $t=x u$ changes these to

$$
\begin{aligned}
\frac{x^{n+2 p+\rho}}{\Gamma(\rho)} \int_{0}^{1}(1-u)^{\rho-1} u^{n+2 p} d u & =\frac{x^{m+2 p}}{\Gamma(\rho)} \cdot B(\rho, n+1+2 p) \\
& =\frac{x^{m+2 p} \Gamma(n+2 p+1)}{\Gamma(n+2 p+1+\rho)}
\end{aligned}
$$


and the $m$ th derivative of one of these terms is

$$
\frac{(m+2 p) ! x^{2 p} \Gamma(n+2 p+1)}{(2 p) ! \Gamma(n+2 p+1+\rho)}
$$

Hence the right member of (4) becomes

$$
\begin{aligned}
\frac{m ! \Gamma(n+1)}{\Gamma(n+1+\rho)} & +\frac{1}{2} \cdot \frac{(m+2) ! \Gamma(n+3)}{2 ! \Gamma(n+3+\rho)} x^{2} \\
& +\frac{1 \cdot 3}{2^{2} \cdot 2 !} \cdot \frac{(m+4) ! \Gamma(n+5)}{4 ! \Gamma(n+5+\rho)} x^{4}+\cdots
\end{aligned}
$$

Since $n+\rho=m$, this reduces to

$$
\begin{aligned}
\Gamma(n+1)\left[1+\frac{1}{2}\right. & \cdot \frac{(n+1)(n+2)}{2 !} x^{2} \\
& \left.+\frac{1 \cdot 3}{2^{2} \cdot 2 !} \cdot \frac{(n+1)(n+2)(n+3)(n+4)}{4 !} x^{4}+\cdots\right],
\end{aligned}
$$

the desired series.

The integration term by term is justified, since $(x-t)^{p-1}$ is integrable and the series which it multiplies is uniformly convergent.

The order of growth of this mean value, as $r$ approaches 1 , is specified by the following theorem.

THEOREM 2.

$$
\begin{aligned}
\lim _{r \rightarrow 1^{-}} & \frac{(1-r)^{n}}{2 \pi} \int_{0}^{2 \pi} P^{n+1}(r, \theta) d \theta \\
& =\frac{(2 m) ! \Gamma(n+1 / 2)}{m ! 2^{m} \Gamma(n+1) \Gamma(n+1 / 2+\rho)}, \quad n>-1 / 2,
\end{aligned}
$$

where $m, n$ and $\rho$ are related as in Theorem 1 .

If $n$ is an integer the right member reduces to $(2 n) ! /(n !)^{2} 2^{n}$.

Proof. Since $\left(1-r^{2}\right)^{2} /\left(1+r^{2}\right)^{2}=1-x^{2}$, where $x$ has the same meaning as in Theorem 1 , and since $(1+r) /\left(1+r^{2}\right)$ will approach unity, it is convenient to replace $(1-r)^{n}$ by $\left(1-x^{2}\right)^{n / 2}$, and prove that the right member of (5) is equal to

$$
\lim _{x \rightarrow 1-}\left(1-x^{2}\right)^{n / 2}\left(1-x^{2}\right)^{(n+1) / 2} \frac{1}{\Gamma(n+1)} \cdot \frac{d^{n}}{d x^{n}}\left(x^{n}\left(1-x^{2}\right)^{-1 / 2}\right) .
$$

If $n=0$, this is obviously true. If $n$ is a positive integer, let 
$\phi(x)=\left(1-x^{2}\right)^{-1 / 2}$, and consider the derivatives of $x^{n} \phi(x)$.

$$
\begin{aligned}
d\left(x^{n} \phi(x)\right) / d x & =x^{n+1} \phi^{3}(x)+n x^{n-1} \phi(x) ; \\
d^{2}\left(x^{n} \phi(x)\right) / d x^{2} & =3 x^{n+2} \phi^{5}(x)+\text { terms in lower powers of } \phi(x) ;
\end{aligned}
$$

and by mathematical induction it can readily be shown that

$$
\begin{aligned}
d^{n}\left(x^{n} \phi(x)\right) / d x^{n}= & 1 \cdot 3 \cdot 5 \cdots(2 n-1) x^{2 n} \phi^{2 n+1}(x) \\
& + \text { terms in } \phi^{2 n-1}, \phi^{2 n-3}, \cdots, \phi .
\end{aligned}
$$

Since

$$
\lim _{x \rightarrow 1-}\left(1-x^{2}\right)^{n+1 / 2} \phi^{p}(x)\left\{\begin{array}{lr}
=0, & p=1,3,5, \cdots, 2 n-1, \\
=1, & p=2 n+1,
\end{array}\right.
$$

the limit in (6) is

$$
\frac{1 \cdot 3 \cdot 5 \cdots(2 n-1)}{\Gamma(n+1)}=\frac{(2 n) !}{2^{n}(n !)^{2}}
$$

as required in the theorem.

If $n$ is not an integer, ${ }^{1}$

$$
\begin{aligned}
\frac{d^{n}}{d x^{n}}\left(x^{n} \phi(x)\right) & =\frac{d^{m}}{d x^{m}} \frac{1}{\Gamma(\rho)} \int_{0}^{x}(x-t)^{\rho-1} t^{n}\left(1-t^{2}\right)^{-1 / 2} d t \\
& =\frac{1}{\Gamma(\rho)} \int_{0}^{x}(x-t)^{\rho-1} \frac{d^{m}}{d t^{m}}\left(t^{n} \phi(t)\right) d t .
\end{aligned}
$$

As before, it is necessary to consider only the first term of the derivative, $1 \cdot 3 \cdot 5 \cdots(2 m-1) x^{m+n} \phi^{2 m+1}(x)$, since

$$
\begin{aligned}
\left(1-x^{2}\right)^{n+1 / 2} \int_{0}^{x}(x-t)^{\rho-1} t^{m+n-2} & \phi^{2 m-1}(t) d t \\
& <\left(1-x^{2}\right)^{n+1 / 2} \int_{0}^{x} \frac{(x-t)^{\rho-1} d t}{\left(1-x^{2}\right)^{m-1 / 2}} \\
& =\left(1-x^{2}\right)^{1-\rho} \int_{0}^{x}(x-t)^{\rho-1} d t,
\end{aligned}
$$

and this approaches zero. Consequently it is necessary to consider

$$
\lim _{x \rightarrow 1-}\left(1-x^{2}\right)^{n+1 / 2} \int_{0}^{x}(x-t)^{\rho-1} t^{m+n} \phi^{2 m+1}(t) d t .
$$

This limit, multiplied by 


$$
\frac{1 \cdot 3 \cdot 5 \cdots(2 m-1)}{\Gamma(\rho) \Gamma(n+1)}
$$

is the result sought.

The substitution $t=x-(1-x) u$ reduces the integral to

$$
\begin{aligned}
& \frac{x^{2 m-\rho}\left(1-x^{2}\right)^{n+1 / 2+\rho}}{(1+x)^{\rho}\left(1-x^{2}\right)^{m+1 / 2}} \\
& \quad \cdot \int_{0}^{x /(1-x)} \frac{u^{\rho-1}(1-(1-x) u / x)^{2 m-\rho} d u}{(1+u)^{m+1 / 2}(1-(1-x) u /(1+x))^{m+1 / 2}} .
\end{aligned}
$$

Since $n+\rho=m$, and $x$ is to approach 1 later, the factor outside the integral sign will have the limit $2^{-\rho}$. To evaluate the integral, let $a$ be a number between 0 and 1 (the way to choose $a$ will become clear later; $a$ will depend on $n$ but not on $x$ ), and consider

$$
\int_{a x /(1-x)}^{x /(1-x)} \text { (same integrand as in (8)) } d u \text {. }
$$

In the interval of integration, $u \geqq a x /(1-x), 1+u \geqq(1-x(1-a)) /(1-x)$, and $1-(1-x) u /(1+x) \geqq 1 /(1+x)$. Hence the integral in (9) is less than

$$
\begin{aligned}
((1-x) / a x)^{1-\rho}(1+x)^{m+1 / 2}\{(1-x) /(1-x(1-a))\}^{m+1 / 2} & \\
\cdot & \int_{a x /(1-x)}^{x /(1-x)}(1-(1-x) u / x)^{2 m-\rho} d u .
\end{aligned}
$$

Except for a bounded factor this is $(1-x)^{m+3 / 2-p} x(1-a)^{2 m+1-p} /(1-x)$, and accordingly approaches zero as $x$ approaches 1 ; if $n$ is negative, $m$ is zero, and $n=-\rho>-1 / 2$.

Therefore the desired limit can be found by replacing the upper limit of integration in $(8)$ by $a x /(1-x)$. This new integral will be not less than

$$
\int_{0}^{a x /(1-x)} \frac{u^{\rho-1}(1-a)^{2 m-\rho}}{(1+u)^{m+1 / 2}} d u .
$$

As $x$ approaches 1 this has the limit $(1-a)^{2 m-\rho} B(\rho, m-\rho+1 / 2)$. Also, the new integral will not be greater than

$$
\begin{aligned}
\int_{0}^{a x /(1-x)} & \frac{u^{\rho-1} d u}{(1+u)^{m+1 / 2}(1-a x /(1+x))^{m+1 / 2}} \\
& =\left(\frac{1+x}{1+x(1-a)}\right)^{m+1 / 2} \int_{0}^{a x /(1-x)} \frac{u^{\rho-1} d u}{(1+u)^{m+1 / 2}}
\end{aligned}
$$


which has the limit $2^{m+1 / 2} /(2-a)^{m+1 / 2} B(\rho, m-\rho+1 / 2)$. If $n$ is negative, $m$ is zero, and the factor $(1-a)^{2 m-\rho}$ will appear in (11) instead of (10).

Since $a$ can be taken as close to zero as is desired, it follows that the limit exists and is $B(\rho, m-\rho+1 / 2)$, or $B(\rho, n+1 / 2)$. If the factor (7) is annexed, the theorem follows.

The theorem cannot be extended to the case $-1<n \leqq-1 / 2$, for if $n$ is replaced by $-\rho$,

$$
\begin{aligned}
\frac{(1-r)^{n}}{2 \pi} \int_{0}^{2 \pi} P^{n+1}(r, \theta) d \theta & \\
& =\frac{(1-r)^{-\rho}}{2 \pi}\left(\frac{1-r^{2}}{1+r^{2}}\right)^{1-\rho} \int_{0}^{2 \pi} \frac{d \theta}{(1-x \cos \theta)^{1-\rho}} .
\end{aligned}
$$

After a bounded factor is removed, the substitution $u=\cos \theta$ gives

$$
(1-x)^{1 / 2-\rho} \int_{-1}^{+1} \frac{d u}{\left(1-u^{2}\right)^{1 / 2}(1-x u)^{1-\rho}} .
$$

Since this integral converges at $u=-1$, it is necessary to consider only the interval from 0 to 1 to show divergence. The change of variable $x u=t(1-x)$ yields

$$
(1-x)^{1 / 2-\rho} \int_{0}^{x /(1-x)} \frac{d t(1-x) / x}{(1-(1-x) t / x)^{1 / 2}(1+(1-x) t / x)^{1 / 2}(1-t(1-x))^{1-\rho}},
$$

which is greater than

$$
\frac{(1-x)^{3 / 2-\rho}}{2^{1 / 2}} \int_{0}^{x /(1-x)}(1-(1-x) t / x)^{-1 / 2} d t=(1-x)^{1 / 2-\rho} 2^{1 / 2} x .
$$

Hence, if $\rho$ is greater than $1 / 2$, the integral diverges and no limit exists.

If $n=-1 / 2$, the theorem fails to hold, but the order of growth of the mean value can be found. Here $m=0, \rho=1 / 2$, and

$$
\begin{aligned}
& \frac{(1-r)^{n}}{2 \pi} \int_{0}^{2 \pi} P^{n+1}(r, \theta) d \theta \\
& \quad=\frac{(1-r)^{-1 / 2}}{2 \pi}\left(\frac{1-r^{2}}{1+r^{2}}\right)^{1-1 / 2} \int_{0}^{2 \pi}(1-x \cos \theta)^{-1 / 2} d \theta .
\end{aligned}
$$

The last integral can be written

$$
\int_{0}^{2 \pi}\left(1-x+2 x \sin ^{2} \theta / 2\right)^{-1 / 2} d \theta
$$


which is greater than

$$
\int_{0}^{2 \pi}\left(1-x+2 \sin ^{2} \theta / 2\right)^{-1 / 2} d \theta
$$

If $1-x$ is set equal to $2 \alpha^{2}$, the last integral is greater than

$$
\frac{1}{2^{1 / 2}} \int_{0}^{2 \pi}\left(\alpha^{2}+\theta^{2}\right)^{-1 / 2} d \theta=\frac{1}{2^{1 / 2}} \log \left(2 \pi+\left(4 \pi^{2}+\alpha^{2}\right)^{1 / 2}\right) / \alpha,
$$

which becomes infinite as $\alpha$ approaches zero. Now $|\log \alpha|$ is effectively $|\log (1-x)|$ or $|\log (1-r)|$ multiplied by a constant. By an estimation similar to the foregoing, the integral can be shown to be less than $|\log (1-r)|$ multiplied by a second constant. Hence, if $n=-1 / 2$, the order of growth of the mean value is $(1-r)^{1 / 2}|\log (1-r)|$.

Colby College and

The University of California at Los Angeles 